\title{
Research on Application of Distributed Optical Fiber Sensing Technology in the Safety Monitoring of Pipeline Transportation
}

\author{
Tao Wang1, a , Binhua $\mathrm{Xu}^{\star 2,3,4, \mathrm{~b}}$, Bin $\mathrm{He}^{2,3, \mathrm{c}}$, Min $\mathrm{Du}^{5, \mathrm{~d}}$ \\ ${ }^{1}$ China Petroleum Pipeline Engineering Corporation, Langfang 065000, China \\ ${ }^{2}$ Geotechnical Engineering Department, Nanjing Hydraulic Research Institute, Nanjing 210029, \\ China \\ ${ }^{3}$ State Key Laboratory of Hydrology-Water Resources and Hydraulic Engineering, Nanjing 210029, \\ China \\ ${ }^{4}$ Hohai University, Nanjing 210098, China \\ ${ }^{5}$ Chongqing Xianglong Natural Gas Co., Ltd., Chongqing 408000, China \\ accppewangtao@cnpc.com.cn, b317413076@qq.com, \\ cbhe@nhri.cn, d52012785@qq.com
}

\begin{abstract}
Keywords: Pipeline transportation; Safety monitoring; Distributed optical fiber sensing technology; Brillouin scattering; Pipeline deformation; Leakage.

Abstract. Pipeline transportation plays a vital role in the transportation of oil and gas. The safety monitoring of pipeline transportation is essential in pipeline operation. Distributed optical fiber sensing technology has more advantages than other conventional pipeline security monitoring method. The ability to measure temperatures and strain at thousands of points along a single fiber is particularly meaningful for the monitoring of pipeline transportation. This paper introduces the principle of distributed optical fiber sensing technology in temperature and strain measurement. DiTeSt (Distributed Temperature and Strain) monitoring system based on Brillouin scattering is used in the monitoring project of Andes pipeline and Italy Rimini gas pipeline for example to detect the geological hazard around pipeline and monitor the deformation and leakage of pipeline. The pipeline monitoring system has been well used in the safety monitoring project of high altitude and long distance pipeline transportation. It shows that DiTeSt(Distributed Temperature and Strain) monitoring system is capable of warning pipeline disasters, improving the efficiency of pipeline transportation safety monitoring, ensuring the normal pipeline transportation safety operation and reducing the property loss of operator.
\end{abstract}

\section{Introduction}

Oil and natural gas are the major traditional energy in the world, providing a powerful impetus for the development of global industry and economy. Pipeline transportation has following features of large capacity: high efficiency, economic space occupation and low cost compared with the traditional transportation method, such as roads and railways. Therefore, pipeline transportation is the most important method of transportation for oil and gas, and it is regarded as one of the top five modes of transportation. Oil and gas pipelines were commonly used to transit dangerous and polluting energy sources, such as oil, natural gas, shale gas and so on. Due to the uneven distribution of energy sources in the most regions, the laying part of the pipeline is generally longer. Many parts of the pipeline are usually located in the wild and even the high attitude area, and the environmental conditions along the pipeline are complex. The deformation of pipes is largely caused by external disturbances and some natural geological disasters while the deformation, corrosion, abrasion, and pipeline defects will further cause pipeline leakage. The leakage of pipeline will not only cause energy waste, but also cause environmental pollution, and even endanger the lives and property of people around the pipeline. The safety monitoring of pipeline transportation is essential in pipeline operation to guarantee the safe operation of pipelines, and it is of great economic, environmental and social 
significance to establish an effective and accurate real-time safety monitoring system of pipeline transportation.

The safety monitoring of pipeline mainly includes deformation monitoring and leakage monitoring. The method of pipeline deformation monitoring includes strain gauge method, ultrasonic method [1], pipeline intelligent sweeper method [2], fiber Bragg grating (FBG) method [3], distributed optical fiber sensing technology and so on. There are many pipeline leakage monitoring methods [4], such as ultrasonic method [5,6], flux leakage detection method[7], distributed optical fiber sensing technology and so on. Distributed optical fiber sensing technology as a new measuring technology has developed rapidly in recent years, it uses optical fiber as the sensor, which can measure temperature and strain at any position. It has the advantages of distributed, continuous and real-time online compared with other traditional pipeline monitoring technology. Fruitful scientific achievements on the application have been achieved in the pipeline safety monitoring project.

This research shows that DiTeSt(Distributed Temperature and Strain) monitoring system which is based on Brillouin scattering principle can implement continuous, on-line and reliable monitoring of pipeline deformation and pipeline leakage in high-altitude and long-distance pipeline transportation projects. This monitoring system can not only improve the management level of pipeline safety operation but also make it efficient.

\section{Monitoring Principle of Distributed Optical Fiber Monitoring System}

\section{The Basic Principle of Brillouin Scattering [8,9]}

Modern optics believes that light has wave-particle duality, and light can be regarded as either an electromagnetic wave or a particle. The light propagates in the optical fiber can causes scattering phenomenon, mainly including Rayleigh scattering, Raman scattering and Brillouin scattering. Rayleigh scattering is caused by the elastic collision of incident light and medium particles, and the scattered light frequency does not shift. Raman scattering is mainly caused by the thermal vibration of fiber molecules. Brillouin scattering is caused by the interaction of photons and phonons in fibers. Both Raman scattering and Brillouin scattering belong to inelastic scattering and the frequency of scattered light is relatively deviates from the incident light frequency. The Raman scattered light has two components of Stokes light and anti-Stokes light, the former has a lower frequency and the latter has a higher frequency. The intensity ratio of Stokes light and anti-Stokes light has a certain functional relationship with the temperature, so temperature monitoring can be implemented by using Raman scattering. The Brillouin scattered light is sensitive to strain and temperature at the same time. When strain and temperature change, Brillouin backscattered light will generate a frequency shift. Strain and temperature measurement can be performed by measuring the Brillouin scattering frequency shift of the pulsed light.

Studies have shown that the Brillouin frequency shift and power have a linear relationship with the strain and the ambient temperature of the fiber under certain conditions. The linear relationships are:

$$
\begin{aligned}
& \Delta v_{B}=C_{v T} \Delta T+C_{v \varepsilon} \Delta \varepsilon \\
& \frac{100 \Delta P_{B}}{P_{B}(T, \varepsilon)}=C_{P T} \Delta T+C_{P \varepsilon} \Delta \varepsilon
\end{aligned}
$$

where $\Delta v_{B}$ is the variation of Brillouin frequency shift, $\Delta T$ is the temperature variation of the fiber, $\Delta \varepsilon$ is the strain variation of the fiber, $C_{v T}$ is the temperature coefficient of Brillouin frequency shift, $C_{v \varepsilon}$ is the strain coefficient of Brillouin frequency shift, $\Delta P_{B}$ is the variation of Brillouin power, $C_{P T}$ is the temperature coefficient of Brillouin power, $C_{P \varepsilon}$ is the strain coefficient of Brillouin power.

With the temperature and strain coefficient, the temperature and strain information can be obtained by the above equation by measuring the frequency shift and power of the Brillouin signal. 


\section{The Sensing Principle of Distributed Optical Fiber Based on Brillouin Scattering}

The current strain and temperature sensing technologies based on Brillouin scattering mainly include Brillouin Optical Time Domain Reflectometer (BOTDR) distributed optical fiber sensing technology and Brillouin Optical Time Domain Analyzer (BOTDR) distributed optical fiber sensing technology[10]. BOTDR and BOTDA technologies enable spatial positioning of measured locations. According to the intensity of incident light (pump light), Brillouin scattering can be divided into self-issuing Brillouin scattering and stimulated Brillouin scattering $(S B S)$. BOTDR technology, based on self-issuing Brillouin scattering principle, requires only a single light source and single-end configuration. On the other hand, BOTDA technology, based on stimulated Brillouin scattering ( $S B S)$ principle, requires dual light sources and loop configuration. Due to the interaction between pulsed light and continuous light, the Brillouin spectrum of the BOTDA technology is amplified so that a higher spatial resolution and accuracy than the BOTDR technology can be obtained $[11,12]$. The Swiss-made DiTeSt monitoring system which is based on the $S B S$ principle of BOTDA technology was used in this research.

\section{DiTeSt(Distributed Temperature and Strain) Monitoring System}

The main components of the DiTeSt monitoring system are software, sensors, and reading units for sensor terminal modules. The reading unit is connected to the proximal end of the sensor and can be placed remotely from the sensing area, since a section of optical fiber cable could be used to link the reading unit to the sensor itself without any performance degradation. The other sensor-end can be either connected to the sensor termination module (single-end configuration), which could be placed remotely from the sensor area as well, or brought back and connected to the reading unit (loop configuration). The selection of the configuration (single-end or loop) depends on the application. The use of optical amplifier modules (range extenders) allows the monitoring of up to $300 \mathrm{~km}$ of pipeline from a single instrument (see Fig. 1). A scheme of the DiTeSt system is given in Fig. 1 and typical performances in Table 1. In the monitoring process, different types of optical fibers can be selected depending on the monitoring requires. There are general temperature sensing optical cables $\left(-40\right.$ to $85^{\circ} \mathrm{C}$ ), extreme temperature sensing optical cables (up to $300^{\circ} \mathrm{C}$ ), strain sensing optical cables (SMARTape) cables and combined strain and temperature sensing optical cables (SMARTprofiles).

Table 1 Performances of DiTeSt system

\begin{tabular}{|c|c|c|c|c|c|c|c|}
\hline $\begin{array}{c}\text { Measurement } \\
\text { range }\end{array}$ & $\begin{array}{c}\text { Number of } \\
\text { channels }\end{array}$ & $\begin{array}{l}\text { Spatial } \\
\text { resolution }\end{array}$ & $\begin{array}{l}\text { Temperature } \\
\text { resolution }\end{array}$ & $\begin{array}{l}\text { Temperature } \\
\text { range } \\
\text { (depends } \\
\text { on type of } \\
\text { sensing } \\
\text { cable) }\end{array}$ & $\begin{array}{l}\text { Strain } \\
\text { resolution }\end{array}$ & $\begin{array}{l}\text { Strain range } \\
\text { (typical) }\end{array}$ & $\begin{array}{c}\text { Acquisition } \\
\text { time } \\
\text { (typical) }\end{array}$ \\
\hline $\begin{array}{c}30 \mathrm{~km} \\
\text { (standard) } \\
150 \mathrm{~km} \\
\text { (extended) }\end{array}$ & 2 (standard) & $\begin{array}{c}1 \mathrm{~m} \text { over } 5 \\
\mathrm{~km} \\
2 \mathrm{~m} \text { over } 25 \\
\mathrm{~km}\end{array}$ & $0.1^{\circ} \mathrm{C}$ & $\begin{array}{l}-40^{\circ} \mathrm{C} \text { to } \\
+300^{\circ} \mathrm{C}\end{array}$ & $\begin{array}{c}2 \mu \varepsilon(0.002 \\
\mathrm{mm} / \mathrm{m})\end{array}$ & $\begin{array}{c}-1.25 \% \text { to } \\
1.25 \%\end{array}$ & 2 minutes \\
\hline
\end{tabular}
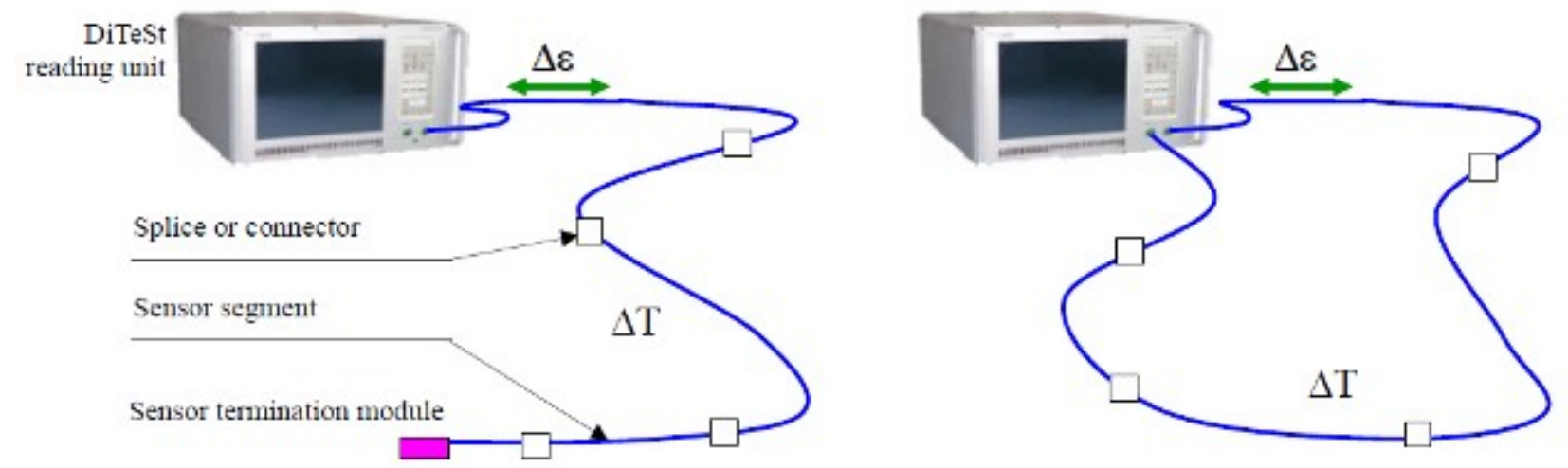

Fig. 1 Scheme of DiTeSt system configurations, left: single-end configuration; right: loop configuration. 
The pipeline will be deformed due to geological structure and geological hazards along the way. Optical fibers will be laid along the pipe or in the soil around the pipe. When the pipe is deformed or the soil around the pipe moves, the fiber will produce strain. The deformation of the pipe and the movement of the soil can be monitored by measuring the strain of the optical fiber. Studies have shown that when the oil and gas pipeline leaks, the temperature field around the pipeline will change greatly $[13,14]$. By monitoring the temperature and strain of the optical fiber sensors installed on the pipeline, it is able to monitor the leakage of the pipeline, soil erosion around the pipeline and other disasters. DiTeSt monitoring system can be used to monitor pipeline safety hazards such as pipeline deformation, soil movement around pipe, pipeline leakage and erosion.

\section{Typical Application Cases}

\section{Pipeline Monitoring across the Andean}

The Trans-Andean Pipeline Monitoring Project is currently the highest altitude pipeline monitoring project in the world. The monitoring pipeline connect the 34"high-pressure liquefied natural gas pipeline operated by Peruvian liquefied natural gas (PLNG) group to the natural gas pipeline operated by Peruvian natural gas pipeline transportation company (TGP). This pipeline extends to the coast $408 \mathrm{~km}$ away, with an altitude of nearly $5000 \mathrm{~m}$ along the pipeline. This line is very challenging for the installation and operation of the pipeline as shown in Fig. 2. First, the geomorphology is so special (a region where landslides and erosion in the region caused more than $50 \%$ of pipeline accidents), and the area has a slope of approximately 45 degrees, requiring effective measures to limit erosion and water runoff. Second, the poor climate conditions, which indicate the switch of hot, dry periods and rainy periods, will easily trigger soil erosion and landslides. Third, in some areas, the change of the water level around the pipeline is also a constant threat.
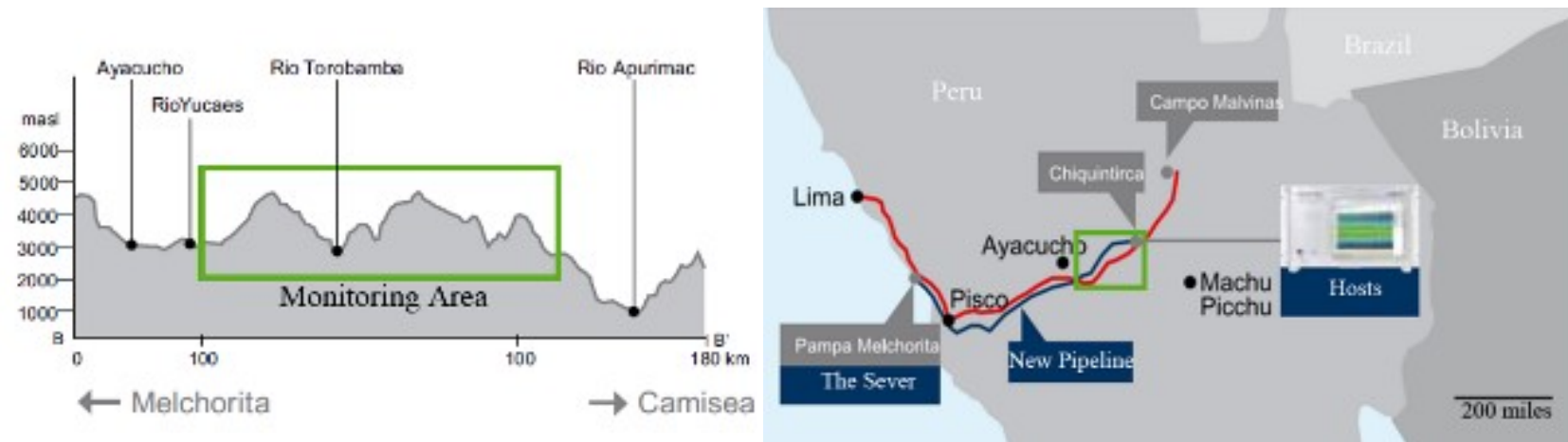

Fig. 2 Sketch map of pipeline

Due to the geological and geomorphological features of the project, strain $g$ and temperature monitoring should be performed around the pipeline. Soil movement may be a sign of pipeline movement, deformation and ultimately fracture, so it requires early detection and accurate positioning, followed by continuous monitoring so that operators can take a solution immediately. The changes of groundwater level and leakage in pipelines can cause the temperature field changed around the pipeline, as a result temperature monitoring needs to be performed around the pipeline. Traditional geotechnical monitoring techniques cannot meet the project's monitoring requirements. Distributed optical fiber sensing system provides the ideal solution for performing deformation and temperature monitoring in these challenging areas. The system is placed at $30 \mathrm{~km}$ away from the first valve station for data processing. The analysis and storage server is located in a control room in Pampa Melchorita, south of Lima. The server can also generate an alert, send it to the SCADA system, and send e-mails to specified recipients (email alert service). Two independent sensing cables are installed around the pipeline. One is a strain gauging cable to accurately reflect the strain caused by the movement of the surrounding soil. The other temperature measuring cable is used for temperature sensing. The strain-sensing optical cable (IEC 794-1 standard) is arranged in the trench to provide early warning of soil movement. Any tension in the strain-sensing optical cable is monitored and located in real-time 
by the DiTeSt system. It will also send an alerts sign when the strain exceeds a user-defined threshold. The cable is sensitive enough to monitor a small soil movement (within $5 \mathrm{~cm}$ ). The temperature information is obtained by means of a temperature-sensing optical cable embedded within the trench at a distance of $10 \mathrm{~cm}$ from the upper surface of the pipe. Temperature events and corresponding position data are sent to the monitoring system. The DiTeSt monitoring system uses automatic leak detection algorithms to monitor water level changes and early leaks. The DiTeSt monitoring system based on the Brillouin scattering principle can monitor soil movements (via strain monitoring) and water level changes (via temperature monitoring) to accurately monitor and locate strain and temperature information for $60 \mathrm{~km}$ pipelines passing through the Andes. At the same time, leakage monitoring provides data and information from continuous temperature monitoring along the pipeline.

Shortly after the pipeline project was run, the system showed an early warning of a sudden increase in strain during a rock fall accident at the position of KP27 +900 meters. The survey found that the rocks in this area had been dropped and the optical fiber sensor in this position had moved, as shown in Fig. 3. Further research showed that the rock fall was caused by an increase in tension cracks on the slope. In order to secure the safety of the pipeline, operators have strengthened this area. In another soil movement accident, the DiTeSt monitoring system alerted a strain incident, which was checked due to seepage (possibly drained from the collapsed water channel). The infiltration resulted in the partly collapse of the pipeline trench, causing a sudden increasing strain in the monitoring system, as shown in Fig. 4. Although the accident did not lead to deformation or damage to the pipeline, the system promptly alerted the movement of the soil so that the operator could repair the trench in time to prevent further damage to the pipeline. During the operation of the pipeline, DiTeSt monitoring system gave early warning of a large number of incidents, all of which are verified through patrol. It facilitates operators to strengthen the pipeline in time to avoid asset losses.
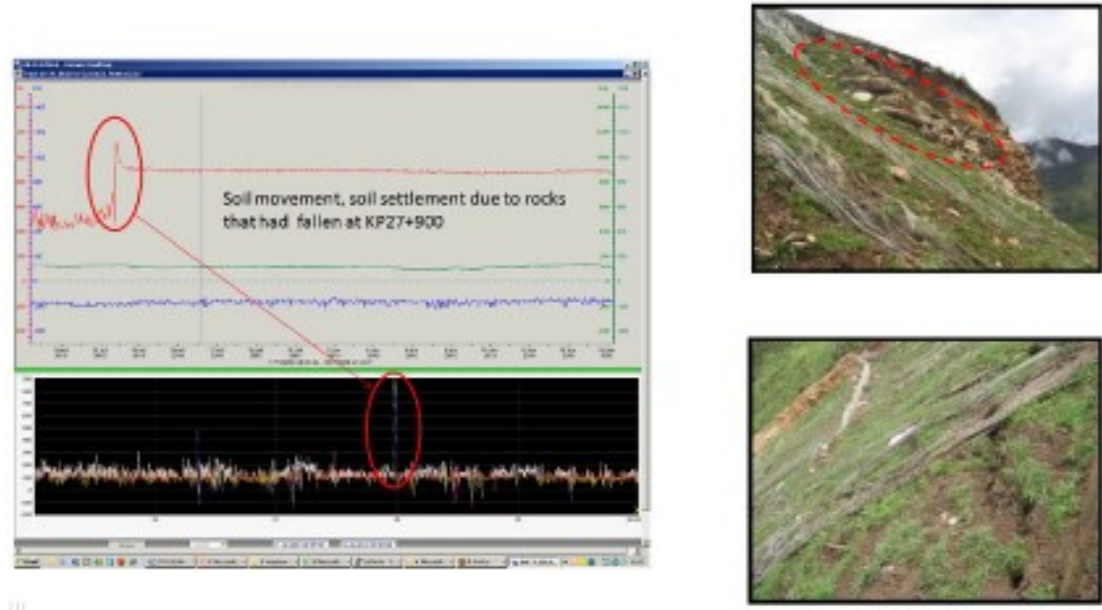

Fig. 3 Monitoring chart and site map of rockfall accident 


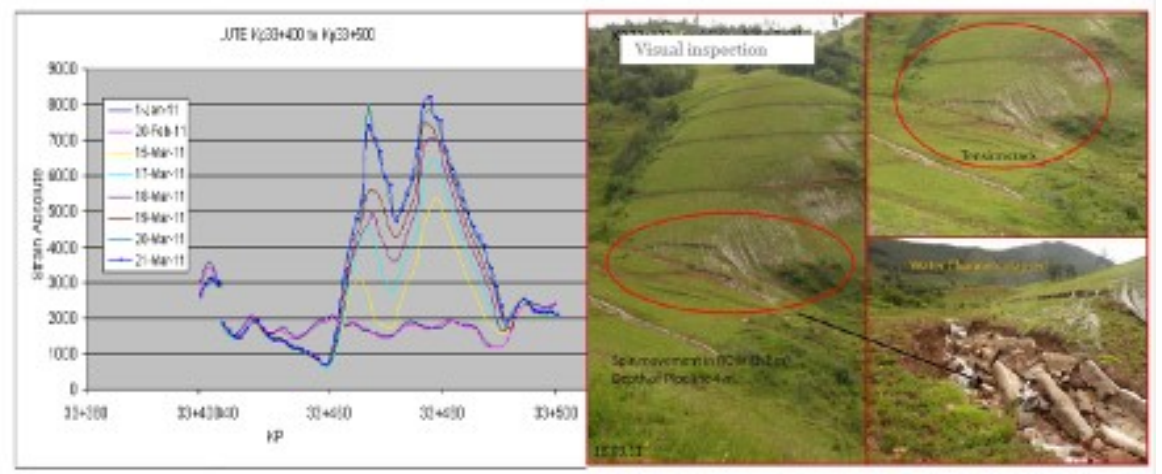

Fig. 4 Monitoring chart and site map of pipeline trench collapse accident

\section{Italian Rimini Gas Pipeline Monitoring Project}

In Rimini, Italy, a 500-meter-long gas pipeline with 35 years old is located in an unstable area. After previous engineering evaluations and calculations, only three symmetrically distributed vibrating wire sensors were installed in the large stress position in the early stage, and the position interval was usually $50 / 100 \mathrm{~m}$. Although these sensors can provide a certain deformation monitoring information, they cannot cover the entire length of the pipeline and provide only partial measurements. In order to improve the monitoring range of existing vibrating wire strain sensors, the monitoring system of the entire pipeline needs to be upgraded so that the system can perform continuous real-time monitoring. Distributed optical fiber sensing technology can meet the strain monitoring requirements of the retrofit project.

The project uses DiTeSt monitoring system, which consists of a host and different types of distributed sensing optical cables (SMARTape and temperature-sensing optical cable). SMARTape sensing cables are installed in the position at approximately $0^{\circ}, 120^{\circ}$, and $-120^{\circ}$ of the pipe axis, with a strain resolution of $20 \mu \varepsilon$ and a spatial resolution of $1.5 \mathrm{~m}$ (sampling interval of $0.25 \mathrm{~m}$ ). SMARTape sensing cables can provide average strain, mean curvature, and pipeline deformation monitoring. The temperature-sensing optical cable is installed at the top of the pipe $\left(0^{\circ}\right)$ to provide temperature compensation for strain measurement. Temperature resolution of the sensor is $1{ }^{\circ} \mathrm{C}$, same as SMARTape's spatial resolution and sampling interval, by using a single DiTeSt host measurement data.

After the re-burial is completed, the pipe will be loaded by the soil and it will be deformed. The diagram showing the strain distribution over all the length of the pipeline after the burring measured by SMARTapes is presented in Fig. 5. The normal cross-sectional strain distribution as well as the curvature distribution in horizontal and vertical plane are calculated from the measured values as shown in Fig. 6. From Fig. 7 and Fig. 8, the strain, strain rate, and bending rate of the pipeline at $50 / 100 \mathrm{~m}$ apart are relatively large. This corresponds to the location of the large stress estimated and calculated in the previous project, indicating that the DiTeSt distributed optical fiber monitoring system can meet the entire pipeline deformation monitoring requirements.

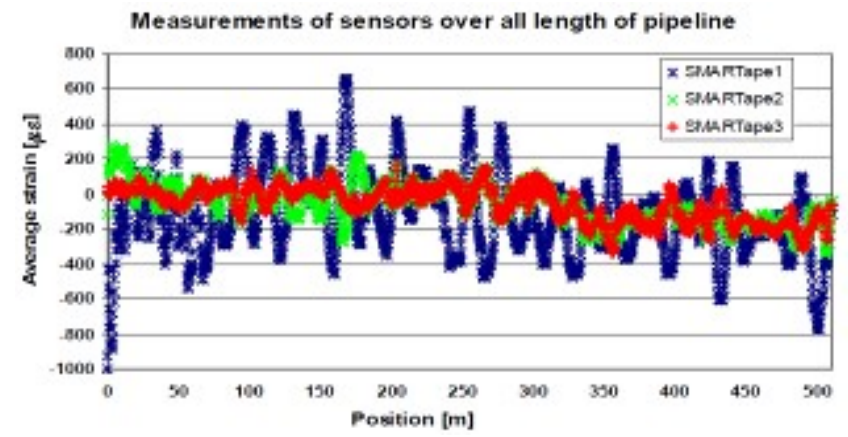

Fig. 5 Monitoring chart of pipeline strain distribution

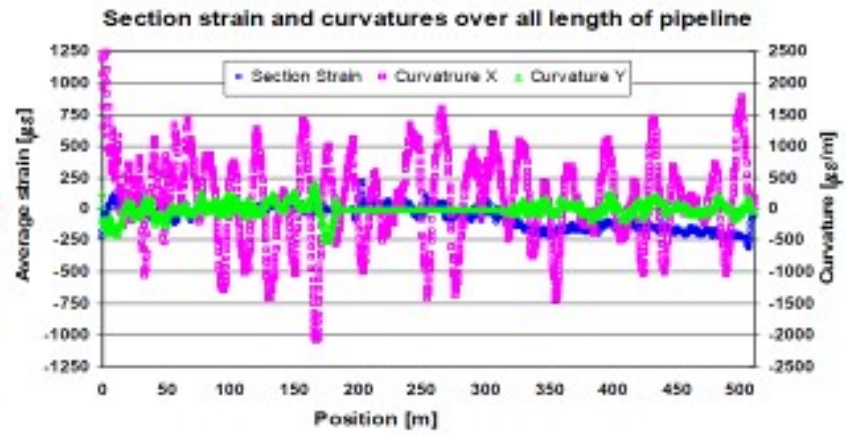

Fig. 6 Monitoring chart of pipeline strain rate and bending rate distribution 


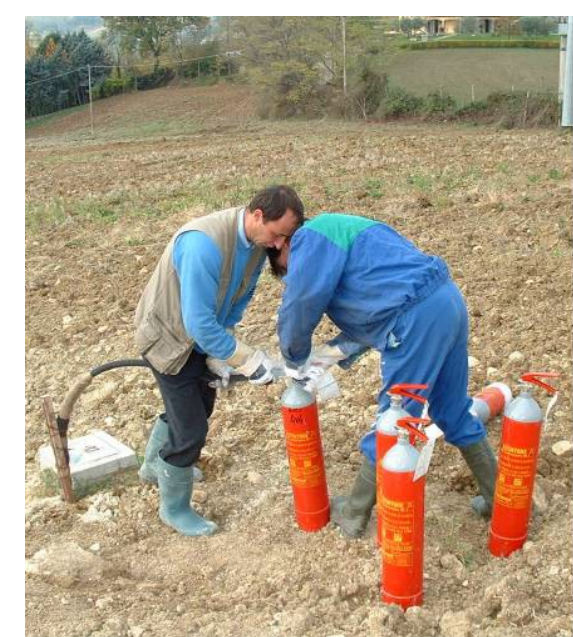

Fig. 7 Field figure of gas leakage simulation

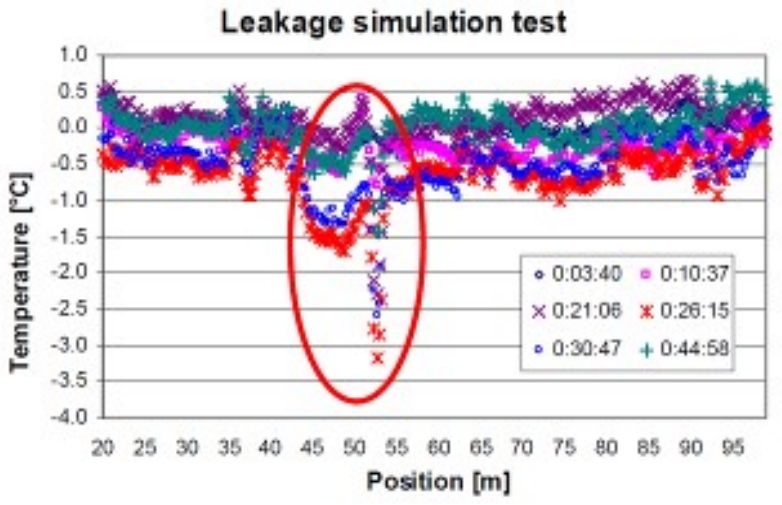

Fig. 8 Monitoring figure of gas leakage simulation

In order to simulate the gas leakage, an empty plastic tube was installed in the upper part of the pipe to connect the pipe top and the ground, as shown in Fig. 7, which was 50m away from the starting point of the first monitoring section. The initial measurement is performed before the plastic tube is cooled. After the carbon dioxide is inserted, a temperature measurement is performed every 2 to 10 minutes and compared with the initial measurement value. The test result is shown in Fig. 8. As can be seen from the figure, the temperature and position information of the simulated leakage point can be accurately displayed in the monitoring system, and the gas leakage point is clearly observed in the chart. It shows that the DiTeSt monitoring system can meet the monitoring requirements for gas leakage of gas pipelines.

\section{Conclusions}

The DiTeSt monitoring system based on Brillouin scattering principle can meet real-time and continuous pipeline monitoring requirements and can be used for pipe safety monitoring such as pipe deformation, soil movement around pipes, pipeline leakage and erosion. The system has been well applied in long-distance high-altitude pipeline safety monitoring projects. It can timely and efficiently perform early warning on disaster events, improving the efficiency of pipeline safety monitoring, ensuring the normal and safe operation of pipelines and reducing the number of operators.

\section{Acknowledgements}

This work was financially supported by the National Key Research and Development Plan of China (2017YFC0405005,Research on Key Technologies and Equipment for Emergency Rescue and Quick Repair of South-to-North Water Transfer Project), National Natural Science Foundation of China (51579152) and Wulingshan Natural Gas Pipeline Project of Distributed Fiber Integrated Monitoring System.

\section{References}

[1] J.J. Xin, J.R. Dong, S.L. Huang, et al: Deformation inspection of petroleum and gas pipeline. Non-detective Testing. Vol. 5 (2008), p. 285-288,294.

[2] F.X. Wang, Q.S. Feng, J.X. Yang, et al: Inertial surveying and application of oil and gas pipelines. Oil \& Gas Storage and Transportation. Vol. 5 (2012), p. 372-375.

[3] J.P. Ou: Research and practice of smart sensor networks and health monitoring systems for civil infrastructures in mainland china. Bulletin of National Natural Science Foundation of China. Vol. 1 (2005), p. 8-12. 
[4] P.S. Murvay, I, Silea: A survey on gas leak detection and localization techniques. Journal of Loss Prevention in the Process Industries. Vol. 6 (2012), p. 966-973.

[5] L.Y. Meng, Y.X. Li, W.C. Wang, et al: Experimental study on leak detection and location for gas pipeline based on acoustic method. Journal of Loss Prevention in the Process Industries. Vol. 1 (2012), p. 90-102.

[6] C.W. Liu, X.J. Li, Y.X. Li, et al: Leak detection and location for natural gas pipelines based on acoustic waves. CIESC Jorunal. Vol. 11 (2014), p. 4633-4642.

[7] J. Jorge, J. Robinson: Risk-based analysis suggests expanding maintenance interval. Pipe Line \& Gas Industry. Vol. 83-3 (2000), p. 33-38.

[8] T.R. Parker, M. Farhadiroushan, V.A. Handerek, et al.: Temperature and strain dependence of the power level and frequency of spontaneous Brillouin scattering in optical fibers. Optics Letters. Vol. 22-11 (1997), p. 787-789.

[9] Y.L. Lin, Y.T. Chen: Design of a Raman scattering distributed optical fiber temperature sensor. Optoelectronic Technology \& Information. Vol. 2 (2002), p. 33-36.

[10]Q.F. Wang, X.G. Qiao, Z.A. Jia, et al: Development of research on Brillouin scattering distributed optic fiber sensing technology. Transducer and Microsystem Technologies. Vol. 7 (2007), p. 7-9.

[11]L.P. Yu, Y.Z. Liu, Z.Y. Dai: Brillouin scattering distributed optical fiber sensor. Laser \& Optoelectronics Progress. Vol. 4 (2006), p. 24-28.

[12]T. Horiguchi, M. Tateda: Optical-fiber-attenuation investigation using stimulated Brillouin scattering between a pulse and a continuous wave. Optics Letters. Vol. 14-8 (1989), p. 408-410.

[13]Z.Y. Wang, Y.X. Li, W.C. Wang, et al: Investigation on the temperature shift from fuel pipeline leakage. Journal of University of Electronic Science and Technology of China. Vol. 2 (2015), p. 306-310.

[14]Q.C. Sun: Application of distributed optical fiber sensor in pipeline leakage. Automation in Petro-chemical Industry. Vol. 5 (2011), p. 54-57. 